\title{
Method of the Year 2010
}

\author{
With the capacity to control cellular behaviors using light and genetically encoded \\ light-sensitive proteins, optogenetics has opened new doors for experimentation \\ across biological fields.
}

The ability to systematically perturb and interrogate cellular behaviors in biology has lagged behind our ability to observe these behaviors. The use of light for perturbation, similarly to its use for measurement, can achieve fast, spatially controlled and minimally invasive modulation of cellular activity.

Although scientists have pursued the idea of using light to control the activity of cells for several decades, the available methods had drawbacks that limited their utility. Optogenetics-our choice of Method of the Year 2010-overcomes many of these drawbacks by combining the use of light and genetically encoded lightsensitive proteins to control the behavior of living cells and organisms. Genetic targeting provides exquisite cell specificity in the intact animal in a manner that cannot be achieved with other photostimulation methods that entirely rely on optical uncaging of signaling molecules, for example.

In recent years, optogenetics has become a booming field thanks to several technological developments. The search for light-sensitive proteins capable of controlling particular cellular behaviors has yielded a diverse set of tools with different functionalities. Progress in the light-delivery technologies, particularly in microscopy, has enabled unprecedented control over in vitro and in vivo illumination. Finally, there have been remarkable improvements both in the strategies to express genes in cells of interest in living organisms as well as in the readout technologies used to measure the biological effects induced by optogenetics.

Optogenetics has already changed the way many neuroscience studies are performed. The technique offers the capacity for multimodal control of neuronal activity in specific cells and at timescales relevant to the brain's in vivo physiology. In recent years, the use of optogenetics in neuroscience studies has shown its potential to unveil the causal relationship between the activity of specific neurons and the behaviors they control. Optogenetics has also contributed to our understanding of the biology that underlies several diseases of the nervous system. With new tools allowing control over a variety of diverse cellular events, optogenetics is now poised to prove its potential in other biological fields as well.

In a Primer (p.24), we outline the basic principles of optogenetics and describe some of the methodol-

ogy used in optogenetic experiments. A news piece (p. 19), focuses on how optogenetics has grown over the years. We also include a short video about optogenetics on our website, where visitors can see the potential of this technique.

Karl Deisseroth describes in a Commentary (p. 26) some of the recent developments in optogenetics and discusses technological advances that the field will be tackling in the future.

Karel Svoboda and Simon Peron discuss how the marrying of optogenetics with sophisticated lightdelivery technologies and genetically encoded reporters of neuronal activity will overcome challenges in the study of brain function (p. 30).

Optogenetics can already be used to control diverse intracellular processes including the localization of proteins to certain parts of the cell or the production of specific second-messenger molecules. Wendell A. Lim and colleagues discuss how the use of these tools to modulate cell signaling cascades will accelerate our understanding of cellular biology (p. 35).

As with many other rapidly evolving technologies, there is still much room for improvement. Peter Hegemann and Andreas Möglich discuss the different strategies that will enrich and improve the optogenetic toolbox (p. 39).

Similarly to previous years, our special feature also includes a selection of Methods to Watch (pp. 43-46), a short list of technologies-necessarily incomplete-that we will be keeping an eye on in the coming years.

The maturing of a technology such as optogenetics, born as an idea in the minds of many scientists and the fruit of multidisciplinary developments, is inevitably a collaborative achievement. In describing this technique and its history, we have necessarily failed to mention many technological developments and research groups that have contributed to the advancement of the field.

Optogenetic technology is increasingly being applied in many laboratories around the world and across disciplines. The possible applications of optogenetics still have to be fully explored. We believe that the transformative effect of using light to modulate defined events in specific cells is on its way to great achievements and has the potential to illuminate unexplored avenues of science. 